\title{
Gametocitos de Plasmodium vivax y Plasmodium falciparum: etapas relegadas en el desarrollo de vacunas
}

\author{
Carla Contreras- 0 choa, M en C, ${ }^{(1)}$ Janine M Ramsey, Ph D.(1)
}

\begin{abstract}
Contreras-Ochoa C, Ramsey JM.
Gametocitos de Plasmodium vivax y Plasmodium falciparum: etapas relegadas en el desarrollo de vacunas.

Salud Publica Mex 2004;46:64-70.

El texto completo en inglés de este artículo está disponible en: http://www.insp.mx/salud/index.html
\end{abstract}

\section{Resumen}

Los gametocitos de Plasmodium son los responsables de la transmisión del huésped vertebrado al mosquito vector. Sufren un proceso de desarrollo complejo a partir de parásitos asexuales, que no está completamente entendido, expresando proteínas y moléculas de adhesión específicas. Son capaces de inducir una respuesta inmune humoral específica con anticuerpos IgG, y celular específica, con producción deTN $F \alpha$, IFN $\gamma$ y proliferación de linfocitos $\gamma \delta^{+}$, aun cuando existen respuestas inducidas en contra de las etapas previas del parásito (esporozoito, exo-eritrocítica y eritrocítica). Las vacunas destinadas a bloquear la transmisión del parásito no contemplan a los gametocitos circulantes en el huésped como blancos de acción, sino que van enfocadas contra antígenos expresados en los gametos y en las etapas posfer tilización. El estudio de los mecanismos que regulan la producción de gametocitos y de la respuesta inmune contra éstos, ofrece una oportunidad para el desarrollo de estrategias adicionales para el control de la transmisión. El texto completo en inglés de este artículo está disponible en: http://www.insp.mx/salud/index.html

Palabras clave: Plasmodium; gametocitos; respuesta inmune; vacunas

\author{
Contreras-Ochoa C, Ramsey JM. \\ Plasmodium vivax and Plasmodium falciparum gametocyte \\ stages are neglected in vaccine development. \\ Salud Publica Mex 2004;46:64-70. \\ The English version of this paper \\ is available at: http://www.insp.mx/salud/index.html
}

\begin{abstract}
A bstract
Plasmodium gametocytes are responsible for transmission from the vertebrate host to the mosquito. Plasmodium gametocytes undergo a complex cycle from asexual stages, through a poorly understood process characterized by expression of stage-specific proteins and adhesion molecules. Gametocytes are capable of inducing specific humoral IgG, and cellular responses, which include induction of TN F $\alpha$, IFN $\gamma$ and $\gamma \delta^{+}$lymphocyte proliferation, in addition to immune responses to other stages of the parasite (spo rozo ite, exo-erythrocytic stages, er ythrocytic stages).Although transmission-blocking vaccines against Plasmodium do not currently include components against the gameto cytes (rather they focus on gametes, zygotes or ookinetes, stages which occur in the mosquito), further understanding of the mechanisms under lying gametocytogenesis and immune responses against these stages may provide additional strategies for more effective transmission inhibition. The English version of this paper is available at: http://www.insp.mx/salud/ index.html
\end{abstract}

Key words: Plasmodium; gametocytes; immune response; vaccines

(1) Dirección de Enfermedades Transmitidas por Vectores, Centro de Investigación sobre Enfermedades Infecciosas, Instituto Nacional de Salud Pública. Cuernavaca, Morelos, México.

Fecha de recibido: 25 de marzo de 2003 - Fecha de aprobado: 4 de septiembre de 2003

Solicitud de sobretiros: D ra. Janine M Ramsey W illoquet. Dirección de Enfermedades Transmitidas por Vectores, Centro de Investigación sobre Enfermedades Infecciosas, Instituto N acional de Salud Pública 62508 Cuernavaca, Morelos, México

Correo electrónico: jramsey@ correo.insp.mx 
E 1 paludismo es una de las principales enfermedades en países tropicales y subtropicales del mundo. De las cuatro especies de parásitos que infectan al humano, Plasmodium falciparum y $P$ vivax son las más importantes desde el punto de vista epidemiológico. El ciclo de vida de este parásito atraviesa varias etapas de desarrollo en el huésped vertebrado (esporozoitos, etapas asexuales exo-eritocíticas e intraeritrocíticas y gametocitos) y otras más en el invertebrado (gametos, zigoto, oocisto, oocineto y esporozoito), cada etapa expresa proteínas específicas, por lo que su estudio se vuelve complejo. ${ }^{1}$

La transmisión de Plasmodium spp. del huésped vertebrado al mosquito vector se lleva a cabo exclusivamente por los gametocitos (precursores de los gametos) y ocurre cuando éstos son ingeridos en una comida de sangre. Los gametocitos permanecen en la circulación del huésped durante todo el curso de la infección, por lo que solamente un paciente con un tratamiento subcurativo o inadecuado permanece infectivo a los mosquitos por periodos prolongados, contribuyendo así a la transmisión de la enfermedad. A pesar de ser la etapa clave para la transmisión existe poca información, en comparación con las etapas asexuales, referente a la biología de los gametocitos y a la respuesta inmune contra ellos, lo que ha ocasionado que estén relegadas en el diseño de estrategias de control. En esta revisión se analizan aspectos básicos del desarrollo de los gametocitos en el huésped vertebrado, así como los avances más recientes sobre inmunidad y diseño de vacunas contra éstos.

\section{El desarrollo de los gametocitos en el huésped vertebrado}

Los gametocitos atraviesan un proceso de desarrollo complejo (gametocitogénesis) que en la actualidad es poco entendido; se desarrollan a partir de parásitos asexuales (merozoitos) dentro del huésped vertebrado y también son intraeritrocíticos, en promedio tardan de 8 a 17 días en madurar a partir de la invasión del merozoito. Se desconoce el estímulo o mensaje responsable de su producción a partir de merozoitos o esquizontes y existen dos hipótesis que tratan de explicarlo. Una de ellas sugiere que los merozoitos ya están comprometidos para desarrollarse hacia formas asexuales o hacia gametocitos antes de la invasión al eritrocito. ${ }^{1}$ La otra hipótesis, por el contrario, sugiere que los merozoitos no están comprometidos en el momento de la invasión a los eritrocitos y que factores ambientales o de "estrés" comprometen a los parásitos a desarrollarse como gametocitos. ${ }^{1,2}$ Del mismo modo, y a pesar de que aún no se conoce el mecanismo exacto que regula este proceso, es interesante saber que en los últimos años el tema de la diferenciación de gametocitos a partir de etapas asexuales ha sido sujeto de un creciente interés y se ha encontrado que algunos factores, principalmente aquellos que inhiben la proliferación de parásitos asexuales, ya sea por mecanismos inmunológicos (la inmunidad del huésped contra la parasitemia asexual se ha correlacionado con un incremento o decremento en la gametocitemia), o por drogas antipalúdicas (pirimetamina y cloroquina) e inclusive mediante hormonas (insulina, progesterona y estradiol) que también estimulan la producción de gametocitos., ${ }^{2,3}$

Independientemente del mecanismo, una vez que la célula recibe la señal para el inicio de la gametocitogénesis, se activan nuevas vías de desarrollo que involucran el cese del ciclo asexual y por la activación de nuevos patrones de transcripción y síntesis de proteínas y glicolípidos; ${ }^{4}$ se ha reportado la síntesis de al menos 26 nuevas proteínas en estas etapas. ${ }^{5}$ En el cuadro I se muestra una lista de las proteínas de gametocitos reportadas hasta el momento, así como su posible función. Paralelo a lo anterior, ocurren cambios en la organización celular acompañados del ensamblaje de filamentos de actina y microtúbulos, lo que ha originado que para fines prácticos, el desarrollo de los gametocitos se divida en cinco etapas (I a V). ${ }^{1}$ Además, los eritrocitos infectados con gametocitos inmaduros expresan ligandos de citoadherencia derivados de los parásitos, permitiendo así que los gametocitos puedan refugiarse en los vasos sanguíneos, $\mathrm{y}$ a medida que estos van madurando se presentan cambios en el perfil de citoadherencia. ${ }^{6}$ Se ha encontrado que los gametocitos inmaduros (etapa I a IV) y los parásitos en etapas asexuales se unen a la molécula CD36 y a otros ligandos, mientras que los gametocitos de etapas maduras (etapa V) no lo hacen, lo que sugiere que hay pérdida del ligando original de citoadherencia de la superficie de gametocitos. ${ }^{1}$ Estudios post mortem en humanos y en modelos animales revelan que el secuestro de gametocitos se da principalmente en el bazo y en médula ósea. ${ }^{7}$ Recientemente se ha encontrado que las moléculas ICAM-1, CD49c, CD166 y CD164 actúan como posibles receptores de células de la médula ósea para la adhesión de los gametocitos. ${ }^{8}$

Una vez dentro del vector, el gametocito macho o microgametocito sufre un proceso de exflagelación y produce de 6 a 8 gametos masculinos móviles, aunque este número es variable dependiendo de la especie, mientras que los gametocitos hembra o macrogametocitos producen solamente un gameto. La gametogénesis ocurre entre 10 a 15 minutos después de la comida de sangre y es el resultado del incremento en la temperatura, el $\mathrm{pH}$ y otros factores desconocidos presentes en el vector. Después de 30 minutos ocurre la fecundación 


\section{Cuadro I \\ Proteínas de gametocitos de Plasmodium vivax y P falciparum}

\begin{tabular}{|c|c|c|c|}
\hline Proteína & Especie & Ubicación & Comentario \\
\hline Pf11-1 & Pf & Vacuola parasitófora de gametocitos & Contribuye a la ruptura del eritrocito durante la gametogénesis ${ }^{52}$ \\
\hline Pf16 & Pf & $\begin{array}{l}\text { Proteína integral de membrana } \\
\text { de gametocitos }\end{array}$ & Contribuye al bloqueo de la transmisión, candidato a vacuna $a^{53}$ \\
\hline Pvs20 & Pv & Gametocitos & Función desconocida ${ }^{54}$ \\
\hline Pvs24 & PV & Gametocitos & Función desconocida ${ }^{54}$ \\
\hline Pf25 & $\mathrm{Pf}$ & $\begin{array}{l}\text { Citoplásmica, se expresa en la } \\
\text { superficie de gametos, zigoto y } \\
\text { ooquineto }\end{array}$ & Contribuye al bloqueo de la transmisión ${ }^{55}$ \\
\hline Pvs25 & Pv & Gametocitos & Contribuye al bloqueo de la transmisión ${ }^{56}$ \\
\hline Pfg27 & Pf & En gametocitos desde etapas tempranas & Contribuye al bloqueo de la transmisión ${ }^{1}$ \\
\hline Pvs28 & $\mathrm{PV}$ & Gametocitos & Contribuye al bloqueo de la transmisión $\mathrm{n}^{56}$ \\
\hline Pv42/37 & Pv & Citoplasma de macrogametocitos & Función desconocida ${ }^{54}$ \\
\hline Pfs48/45 & Pv & $\begin{array}{l}\text { Citoplasma de gametocitos y en la } \\
\text { superficie de gametos }\end{array}$ & Contribuye al bloqueo de la transmisión ${ }^{1}$ \\
\hline $\begin{array}{l}\text { Alfa tubulina II } \\
50 \mathrm{kDa}\end{array}$ & PV & Axonema de microgametocitos & $\begin{array}{l}\text { Participa en los cambios morfológicos durante la exflagelación y en la } \\
\text { motilidad del parásito } 57\end{array}$ \\
\hline Pvs57 & $\mathrm{PV}$ & Gametocitos & Función desconocida ${ }^{54}$ \\
\hline Pfs230 & Pf & Superficie de gametocitos y gametos & Contribuye al bloqueo de la transmisión ${ }^{58}$ \\
\hline PfEMP-1 & Pf & Gametocitos desde etapas tempranas & Contribuye a la producción de gameto citos, regulando su maduración ${ }^{59}$ \\
\hline Pfg377 & Pf & Macrogametocitos maduros & Función desconocida ${ }^{60}$ \\
\hline PSLAP & Pf & Gametocitos maduros & $\begin{array}{l}\text { Participa en la modulación y protección contra el sistema inmune del } \\
\text { mosquito } 61\end{array}$ \\
\hline
\end{tabular}

y la subsiguiente transformación del zigoto en un oocineto móvil, el cual penetra la pared del estómago del mosquito enquistándose y se transforma en oocisto. Después de 8 a 15 días, dependiendo de la especie de Plasmodium, el oocisto maduro libera cientos de esporozoitos que invaden las glándulas salivales del mosquito y son inoculados al huésped vertebrado en cada comida de sangre. $3,5,9$

En contraste con la gran cantidad de información referente a la respuesta inmune del huésped contra las etapas asexuales del parásito, se sabe muy poco acerca de la respuesta humoral y celular contra los gametocitos, debido, en parte, a que no se multiplican, ni causan síntomas al humano. Los gametocitos constituyen además la última etapa de desarrollo en el huésped vertebrado y por lo tanto se desarrollan en un ambiente inmune policlonal estimulado por todas las etapas asexuales anteriores (esporozoitos, etapas asexuales exo-eritocíticas e intraeritrocíticas). ${ }^{10}$ Aunado a lo anterior, está el hecho de que pueden permanecer infectivos a los mosquitos durante todo el curso de la infección (excepto durante los paroxismos febriles), lo que sugiere que la respuesta inmune del huésped contra éstos es ineficiente. ${ }^{11}$

\section{Respuesta humoral}

Los gametocitos son capaces de estimular una respuesta humoral específica, sin embargo, por el hecho de que son intraeritrocíticos esta respuesta es limitada. Una vez que las membranas del eritrocito se rompen, las estructuras internas de los gametocitos son altamente inmunogénicas. ${ }^{12}$ En poblaciones humanas infectadas por vía natural con $P$ falciparum se han detectado anticuerpos de los subtipos IgG1 y/o IgG3 contra las proteínas Pfs230 y Pfs48/45 presentes en forma de mensajero citoplásmico durante la etapa de gametocitos, y que se expresan más tarde en la superficie de los gametos. ${ }^{13}$ La proteína Pfs 230 es el blanco principal de anticuerpos que fijan complemento y está correlacionada con la inhibición de la infectividad de los gametocitos a los mosquitos. ${ }^{14}$ Se ha detectado, además, que anticuerpos del subtipo IgG obtenidos de suero hiperinmune de pacientes reconocen la superficie de gametocitos de etapas tempranas (etapa I a II-A) pero no de etapas posteriores. ${ }^{4}$ Del mismo modo, se ha reportado también la presencia de anticuerpos contra la proteína Pf11-1 que participa en el proceso de ruptura de los eritrocitos durante la gametogénesis. ${ }^{15}$ 
En infecciones con $P$ vivax ocurre un proceso similar; la mayoría de los pacientes durante la etapa aguda desarrollan una inmunidad humoral que también suprime la infectividad de los gametocitos a los mosquitos. ${ }^{16}$ En un estudio en Sri Lanka se encontró que el suero de $48 \%$ de los pacientes tenía actividad supresora. ${ }^{17}$ Mientras que en México se encontró que mosquitos An albimanus, que fueron alimentados con eritrocitos infectados con $P$ vivax en presencia de suero autólogo de pacientes de infección primaria o secundaria, manifestaron una inmunidad bloqueadora de la transmisión, siendo mayor en el último grupo de pacientes. ${ }^{18}$

\section{Respuesta celular}

Durante la etapa aguda de la infección con $P$ vivax se han detectado niveles elevados de factor necrosante tumoral alfa (TNF $\alpha$ ) y de interferón gamma (IFN $\gamma$ ) en el suero de pacientes, fenómeno que coincide con la ruptura de parásitos asexuales en etapa de esquizontes. ${ }^{19}$ Del mismo modo, se ha encontrado que durante esta etapa hay una disminución en la infectividad de los gametocitos asociada con un decremento en el número de microgametocitos, pero no de macrogametocitos, circulando en sangre, ${ }^{18}$ el cual se ha relacionado con el incremento de estas citocinas y con otros factores presentes en el suero aún no identificados. ${ }^{20}$

Tal como se esperaría, se ha detectado la proliferación de células mononucleares circulantes (CMC) en respuesta a los gametocitos de $P$ vivax y $P$ falciparum. Sin embargo, la mayoría de estos estudios in vitro se han llevado a cabo en CMC de donadores no palúdi$\cos$. Se ha reportado que las clonas de linfocitos T que reaccionan contra los gametos de $P$ falciparum también reaccionan contra esquizontes, pero no con eritrocitos no infectados, lo que podría sugerir que ambas etapas comparten epítopes comunes. ${ }^{21}$ Por otro lado, se ha demostrado que los gametocitos de $P$ falciparum activan a linfocitos $\mathrm{CD}^{+}$, pero no a linfocitos $\gamma \delta^{+} .22$ En poblaciones humanas infectadas de manera natural con $P$ falciparum y $P$ vivax también se han detectado respuestas proliferativas, específicas y no específicas, dirigidas contra los gametocitos. ${ }^{23,24}$ En ensayos in vitro se encontró proliferación y producción de IFN $\gamma$ en CMC de pacientes infectados con $P$ falciparum estimuladas con gametocitos lisados, estas mismas células también proliferaron al ser estimuladas con la proteína Pf48/45 pura, mientras que en los controles no palúdicos la respuesta proliferativa fue baja. ${ }^{23,25}$

Durante su desarrollo dentro del eritrocito el Plasmodium secreta al medio extracelular una clase particular de proteínas conocidas como exoantígenos o antígenos solubles. Muchas de estas proteínas han sido identifica- das como moléculas clave, involucradas en procesos metabólicos y en la estimulación de la respuesta humoral y celular contra el parásito. ${ }^{26}$ La mayoría de los exoantígenos reportados en la literatura corresponden a etapas asexuales del parásito y son capaces de inducir proliferación de linfocitos y producción de IFNg. ${ }^{27,28}$ Ramsey y colaboradores detectaron, por primera vez, la presencia de exoantígenos secretados específicamente por los gametocitos de $P$ vivax y $P$ falciparum. Estos exoantígenos, de entre 50 y $100 \mathrm{kDa}$, estimularon de manera significativa la proliferación de linfocitos $\gamma \delta^{+} \mathrm{y}$ de forma accesoria la expansión de linfocitos $\mathrm{CD}^{+}$en pacientes con infección primaria o secundaria previa, pero no en los controles no palúdicos, mientras que la expansión de linfocitos $\alpha \beta^{+}$y CD4 $4^{+}$fue mínima. ${ }^{29}$

Los conocimientos obtenidos de este trabajo con gametocitos y de los estudios in vitro contra las etapas asexuales del parásito en donadores no palúdicos, ${ }^{30-33}$ aunado a la expansión de los linfocitos $\gamma \delta^{+}$durante la fase aguda de la enfermedad, encontrada en donadores de áreas endémicas, ${ }^{34,35}$ ponen de manifiesto la importancia de estas células en la respuesta inmune contra Plasmodium. Todas estas evidencias indican que los linfocitos $\gamma \delta^{+}$participan directamente en la eliminación del parásito, en colaboración con otras poblaciones celulares accesorias, y que contribuyen, además, a la regulación de la respuesta inmune contra la infección mediante la producción de citocinas inflamatorias, como TNF $\alpha$ y $\beta$, IFN $\gamma$, IL-5, IL-6, IL-8 y linfotoxina. ${ }^{36,37}$ Sin embargo, el mecanismo de acción y regulación de estas células, así como la naturaleza de los antígenos que reconocen, no están dilucidados todavía, por lo que será necesario que la investigación actual ponga un mayor énfasis en caracterizar esta respuesta.

Cabría aquí mencionar que, en general, existe poca información sobre la biología de los linfocitos $\gamma \delta^{+}$, ya que su descubrimiento es relativamente reciente. Se sabe que se encuentran en todos los organismos con sistema linfoide y que constituyen una población celular minoritaria, de 1 a $5 \%$ del total de linfocitos circulantes en sangre, mientras que en tejidos como el epitelio y mucosas, su número es más abundante. ${ }^{38}$ Además, hay evidencias que señalan que hay varios subtipos y que llevan a cabo funciones diferentes dependiendo del tejido en el que se encuentren. ${ }^{38-40}$

Resulta bastante interesante el hecho de que, a pesar de su reducido número, los linfocitos $\gamma \delta^{+}$tienen un papel determinante en la respuesta inmune contra microorganismos patógenos además de Plasmodium, tales como Mycobacterium tuberculosis, ${ }^{41,42}$ Toxoplasma gondii, ${ }^{43}$ Leishmania donovan ${ }^{44}$ y Mesocestoides corti, causante de la cisticercosis. ${ }^{45}$ Además, en infecciones por virus como el $\mathrm{VIH}^{46}$ herpes, vaccinia, estomatitis ve- 
sicular e influenza, estos linfocitos se activan y migran a los sitios de replicación viral. ${ }^{47} \mathrm{~A}$ su vez, existen reportes que indican que manifiestan actividades antitumorales, monitoreando y eliminando a células transformadas. ${ }^{48}$ La evidencia creciente indica que los linfocitos $\gamma \delta^{+}$ llevan a cabo estas funciones debido a que presentan una actividad citotóxica potente en respuesta al antígeno y a que secretan citocinas que activan a otros mecanismos y poblaciones celulares, potenciando la respuesta inmune..$^{36,39,41}$ Se ha reportado también que no requieren de células presentadoras, ni del procesamiento del antígeno, tal como los linfocitos $\alpha \beta^{+} 1$ o hacen, lo que estaría permitiendo respuestas directas y muy rápidas contra el antígeno. ${ }^{38,39}$

\section{Vacunas contra gametocitos}

Como se ha señalado, debido a la diversidad de etapas y a la complejidad del ciclo de vida del Plasmodium, la estrategia a seguir para el desarrollo de vacunas antipalúdicas ha sido atacar el problema empleando diversos enfoques. Se han diseñado vacunas contra $P$ falciparum y $P$ vivax destinadas a: a) prevenir la infección al humano y van dirigidas contra los esporozoitos y las etapas hepáticas; b) encaminadas a detener la propagación del parásito en la sangre del huésped y van enfocadas contra las etapas asexuales intraeritrocíticas; c) destinadas a reducir o interrumpir la transmisión del parásito del huésped humano al mosquito y van dirigidas contra las etapas sexuales del parásito (gametocitos, gametos, zigoto y etapas esporogónicas en el vector), y se conocen como vacunas para el bloqueo de la transmisión $(\mathrm{VBT})^{49}$, y d) las vacunas anti-enfermedad, también conocidas como antitóxicas, destinadas a disminuir o inhibir algunas de las manifestaciones clínicas de la enfermedad, como la fiebre, que se originan como consecuencia de la respuesta inflamatoria del huésped (mediada por citocinas como el TNF $\alpha$ e Interleucinas IL-1, IL-4, IL-6) en contra de los exoantígenos y moléculas tóxicas derivadas del metabolismo del parásito. 11,14,49-51

La estrategia actual de las VBT consiste en la inducción de una respuesta humoral en el huésped vertebrado, mediante anticuerpos dirigidos contra antígenos de las proteínas de superficie de los gametocitos o gametos. Estos anticuerpos estarían actuando en el intestino del mosquito de 5 a 10 minutos después de la ingestión de sangre, previniendo con esto la fertilización, inhibiendo el reconocimiento celular entre gametos o bien, destruyendo los gametos o los zigotos recién fertilizados, mediante reacciones mediadas por complemento. ${ }^{11} \mathrm{~A}$ este tipo de vacunas se les conoce también como "vacunas altruistas", ya que no confieren protección directa al individuo donador sino que más bien inhiben la transmisión a otro huésped. Debido a que no se interrumpe la trasmisión completamente, este tipo de vacuna podría ser utilizada en áreas de baja incidencia contribuyendo a reducir la transmisión por abajo del umbral crítico requerido para mantener la población de mosquitos infectados, o bien como componente de una vacuna múltiple usada en combinación con componentes parasitarios de etapas asexuales en áreas de alta trasmisión, orientada no sólo al control sino también a la erradicación del parásito. ${ }^{50,51}$ Hasta el momento los antígenos blanco empleados en el desarrollo de VBT se dividen en dos grupos: el primero de ellos comprende los antígenos pre-fertilización, los cuales se pueden encontrar en forma de RNA mensajero o de proteína en los gametocitos y en los gametos, ejemplos de estos antígenos se enlistan en el cuadro I. Dos de las proteínas más ampliamente estudiadas son la Pfs $48 / 45$ y Pfs230 expresadas en $P$ falciparum, anticuerpos monoclonales contra estas proteínas bloquean la infectividad de los gametocitos a los mosquitos, la primera es independiente del complemento y la segunda depende de él. ${ }^{11}$ El segundo grupo comprende antígenos posfertilización expresados en las etapas de zigoto y ooquinetos, los cuales bloquean el desarrollo de éstos y pueden interferir en el desarrollo normal de los esporozoitos. ${ }^{11,62}$

Como se ha expuesto, el estudio de los gametocitos ha estado relegado debido, por un lado, a que no están implicados en la patología de la enfermedad y por el otro, a las dificultades técnicas y metodológicas que implica la obtención de gametocitos puros para los ensayos in vitro. Esto ha provocado una carencia de conocimientos sobre los mecanismos moleculares que permiten la diferenciación sexual a partir de etapas asexuales, además de los mecanismos de modulación de los gametocitos en la circulación y aún más importante, del papel que desempeña la inmunidad humoral y celular en el bloqueo de la transmisión del parásito. Esta desinformación conduce a que las estrategias actuales para el desarrollo de vacunas no contemplen a los gametocitos intraeritrocíticos residentes en la circulación del huésped humano como posibles blancos, sino que se han enfocado principalmente en la etapa de gametos y en las posteriores etapas posfertilización.

El interés creciente en el estudio de la respuesta inmune contra las proteínas gametocitarias ofrece un campo potencial para el desarrollo de estrategias que permitan la eliminación de los gametocitos directamente en la circulación del huésped, disminuyendo con esto la infectividad y/o el número de gametocitos circulantes en el momento cuando el mosquito ingiere la comida de sangre y permitiría, en combinación con estrategias alternas, bloquear la transmisión del parásito. 


\section{Referencias}

1. Lobo CA, Kumar N. Sexual differentiation and development in the malaria parasite. Parasitol Today 1998;14(4):146-150.

2. Dier M, D ay KP. Commitment to gametocytogenesis in Plasmodium falciparum. Parasitol Today 2000;16(3):102-107.

3. Paul R, Brey P, Robert V. Plasmodium sex determination and transmission to mosquitoes. Trends Parasitol 2002;18(1):32-38. 4. Day K, H ayward R, Smith D, C ulvenor J. CD-36 dependent adhesion and knob expression of the transmission stages of Plasmodium falciparum is stage specific. Mol Biochem Parasitol 1998;93(2):166-177. 5. Sinden RE. Sexual development of malarial parasites. Adv Parasitol 1983;22:153-216.

6. Rogers N , Daramola O, Targett G, Hall B. CD 36 and intercellular adhesion molecule mediate adhesion of developing Plasmodium falciparum gametocytes. Infect Immun 1996;64(4):1480-1483. 7. Smalley ME,Abdalla S, Brown J.The distribution of Plasmodium falciparum in the peripheral blood and bone marrow of $\mathrm{G}$ ambian children.Trans R Soc Trop Med Hyg 1981;75(1):103-105. 8. Rogers N , Hall B, O biero J, Targett G, Sutherland C. A model for sequestration of the trasmission stages of Plasmodium falciparum: Adhesion of gametocyte-infected erythrocytes to human bone marrow cells. Infect Immun 2000;68(6):3455-3462.

9. Sinden RE, Butcher GA, Billker 0, Fleck SL. Regulation of infectivity of Plasmodium to the mosquito vector. Adv Parasitol 1996;38:53-117.

10. Mendis KN , D avid PH, Carter R. Human immune responses against sexual stages of malaria parasites: $C$ onsiderations for malaria vaccines. Int J Parasitol 1990;20(4):497-502.

11. Carter R.Transmission blocking malaria vaccines.Vaccine 2001;19(17-19):2309-2314.

12. Miller LH, Hoffman SL. Research toward vaccines against malaria. $N$ at Med 1998;4(5 Suppl):520-524.

13. Healer JD, MCG uinness P, Hopcroft S, Haley R, C arter C, Riley E. Complement-mediated lysis of Plasmodium falciparum gametes by malaria-immune human sera is associated with antibodies to the gamete surface antigen Pfs230. Infect Immun 1997;65(8):3017-3023.

14. Kwiatkowski D, Marsh K. D evelopment of a malaria vaccine. Lancet 1997;350(9092): 1696-1701.

15. Scherf A, Carter R, Petersen C,A lano P, N elson R, Aikawa M et al. $G$ ene activation of Pf11-1 of Plasmodium falciparum by chromosome breakage and healing: Identification of gametocyte-specific protein with a potential role in gametogenesis. EMBO J 1992;11(6):2293-2301. 16. Mendis K, Munesinnghe Y, de Silva Y, Keragalla I, C arter R. Malaria transmission-blocking immunity induced by natural infections of Plasmodium vivax in humans. Infect Immun 1987;55(2):369-372. 17. G amage-Mendis AC, Rajakaruna, Carter R, Mendis KN . Transmission blocking immunity to human Plasmodium vivax malaria in an endemic population in Kataragama, Sri Lanka. Parasite Immunol 1992;14(4):385-396. 18. Ramsey J, Salinas E, Rodríguez MH . A cquired transmission-blocking immunity to Plasmodium vivax in a human population of Southern coastal Mexico.Am J Trop Med Hyg 1996;54(5):458-563.

19. N aonatune T, Karunaweera N D, D el Giudice G, Kularatne MU, G rau $\mathrm{GE}, \mathrm{C}$ arter $\mathrm{R}$ et al. Cytokines kill malaria parasites during infection crisis: Extracellular complementary factors are essential.J Exp Med 1991;173(3):523-529.

20. Karunaweera N, C arter R, Grau G, Kniatkowski D, D el Guidice G, Mendis K.Tumour necrosis factor-dependent parasite-killing effects during paroxysm in non-immmune Plasmodium vivax malarial patients. Clin Exp Immunol 1992;88(3):499-505.

21. Good MF, Q uakyi A, Berzofsky R, C arter R, Miller L. Human cell clones reactive to the sexual stages of Plasmodium falciparum malaria:
High frequency of gamete-reactive T cells in peripheral blood from nonexposed donors. J Immunol 1987;138(1):306-311.

22. Goodier M,Targett G. Polyclonal T-cell responses to Plasmodium falciparum gametocytes in malaria nonexposed donors. Parasite Immunol 1997;19(9):419-425.

23. Riley $\mathrm{E}, \mathrm{O}$ ng $\mathrm{C}, \mathrm{O}$ lerup $\mathrm{O}$, Eida $\mathrm{S}, \mathrm{A}$ llen $\mathrm{S}$, Bennett $\mathrm{S}$ et al. C ellular and humoral immune responses to Plasmodium falciparum gametocyte in malaria-immune individuals. J Immunol 1990;144(12):4810-4816. 24. Riley EM, Allen SJ, W heeler J, Blackman M, Bennett S, Takacs B et al. $N$ aturally acquired cellular and humoral responses to the major merozoite surface antigen (PFMSP1) of Plasmodium falciparum are associated with reduced malaria morbidity. Parasite Immunol 1992;14(3):321-337.

25. Baird JK, Jones TR, D anudirgo EW, Annis BA, Bangs MJ, Basri $\mathrm{H}$ et al. Age-dependent acquired protection against Plasmodium falciparum in people having two years exposure to hyperendemic malaria. Am J Trop Med Hyg 1991;45(1):65-76.

26. Jakobsen PH. Plasmodium falciparum malaria parasite exoantigens: Their role in disease and in immunity. D an Med Bull 1995;42(1):22-39. 27. Luty AJ, Mayombo J, Lekoulou F, M shana R. Immunologic responses to soluble exoantigens of Plasmodium falciparum in $\mathrm{G}$ abonese children exposed to continuous intense infection. Am J Trop Med Hyg 1994;51(6):720-729.

28. Kristensen $\mathrm{G}$, Jakobsen PH. Plasmodium falciparum: C haracterization of toxin-associated proteins and identification of a hemoglobin containing parasite cytokine stimulator. Exp Parasitol 1996;82(2):147-154.

29. Ramsey JM, Tello A, Contreras C O, O rdóñez R, Chirino N, Rojo J et al. Plasmodium falciparum and P. vivax gametocyte-specific exoantigens stimulate proliferation of TCR gd ${ }^{+}$lymphocytes. J Parasitol 2002;88(1):59-68.

30. Behr C, Poupot R, Peyrat MA, Poquet Y, C onstant P, D ubois P et al. Plasmodium falciparum stimuli for human gamma delta $T$ cells are related to phosphorylated antigens of mycobacteria. Infect Immun 1996:64(8):2892-2896 .

31. Pichyangkul S, Saengkrai P,Yongvanitchit K, Stewart A, Heppner DG. Activation of gamma delta T cells in malaria: Interaction of cytokines and a schizont-associated Plasmodium falciparum antigen. Infect $D$ is 1997;176(1):233-241.

32.W aterfall M, Black A, Riley E. Gamma delta+T cells preferentially respond to live rather than killed malaria parasites. Infect Immun 1998;66(5):2393-2398.

33. Hensmann M, K wiatkowski D. C ellular basis of early cytokine response to Plasmodium falciparum. Infect Immun 2001;69(4):2364-2371. 34.W orku S, Bjorkman A, Troye-Blomberg M, Jemaneh L, Farnert A, Christensson B. Lymphocyte activation and subset redistribution in the peripheral blood in acute malaria illness: $D$ istinct gamma delta+T cell patterns in Plasmodium falciparum and P. vivax infections. C lin Exp Immunol 1997;108(1):34-41.

35. H viid L, Kurtzhals JA,A dabayeri V, Loizon S, Kemp K, Goka BQ et al. Perturbation and proinflammatory type activation of $V$ delta $1(+)$ gamma delta T cells in African children with Plasmodium falciparum malaria. Infect Immun 2001;69(5):3190-3196.

36. Rzepczyk CM,Anderson K, Stamatiou S,Townsend E,Allworth A, McC ormack J et al. Gamma deltaT cells:Their immunobiology and role in malaria infections. Int J Parasitol 1997;27(2):191-200.

37.Troye-Blomberg $M$, W orku S,Tangteerawatana $P$, Jamshaid $R$, Soderstrom K, Elghazali $G$ et al. Human gamma deltaT cells that inhibit the in vitro growth of the asexual blood stages of the Plasmodium falciparum parasite express cytolytic and proinflammatory molecules. Scand J Immunol 1999;50(6):642-650.

38. Carding SR, Egan PJ. Gamma deltaT cells: Functional plasticity and heterogeneity. N ature Rev Immunol 2002;2(5):336-345. 
39. Chien YH, Jores R, Crowley MP. Recognition by gamma/delta T cells. Annu Rev Immunol 1996;14:511-532.

40. Chen ZW. Comparative biology of gamma delta T cells. Sci Prog 2002;85(Pt 4):347-358.

41. Dieli F, Troye-Blomberg M, Ivanyi J, Fournie J], Bonneville M, Peyrat MA et al. Vgamma9/Vdelta2 T lymphocytes reduce the viability of intracellular Mycobacterium tuberculosis. Eur J Immunol 2000;30(5):1512-1519.

42. Ferrero E, Biswas P,Vettoretto K, Ferrarini M, U guccioni M, Piali L et al. Macrophages exposed to Mycobacterium tuberculosis release chemokines able to recruit selected leucocyte subpopulations: Focus on gamma delta cells. Immunology 2003;108(3):365-374.

43. N akano Y, H isaeda H, Sakai T, Ishikawa H, Z hang M, Maekawa Y et al. Roles of N KT cells in resistance against infection with Toxoplasma gondii and in expression of heat shock protein 65 in the host macrophages. Microbes Infect 2002;4(1):1-11.

44. Lagler $\mathrm{H}, \mathrm{W}$ illheim M, Traunmuller $\mathrm{F}, \mathrm{W}$ ahl $\mathrm{K}$, W inkler $\mathrm{H}$, Ramharter $M$ et al. C ellular profile of cytokine production in a patient with visceral leishmaniasis: Gamma delta+T cells express both type 1 cytokines and interleukin-10. Scand J Immunol 2003;57(3):291-295.

45. Cardona AE, G onzález PA, Teale JM. CC chemokines mediate leukocyte trafficking into the central nervous system during murine neurocysticercosis: Role of gamma deltaT cells in amplification of the host immune response. Infect Immun 2003;71(5):2634-2642.

46. Poccia F, Gougeon ML, A grati C, Montesano C, Martini F, Pauza CD et al. Innate T-cell immunity in HIV infection:The role of Vgamma9Vdelta2 T lymphocytes. Curr Mol Med 2002;2(8):769-781. 47. Sciammas R, Bluestone JA.TCR gamma delta cells and viruses. Microbes Infect 1999;1(3):203-212.

48. López RD. Human gamma delta-T cells in adoptive immunotherapy of malignant and infectious diseases. Immunol Res 2002;26(1-3):207-221. 49. Playfair JH, Taverne J, Bate CA. D on't kill the parasite: Control the disease.Acta Leiden 1991;60(1):157-165.

50. Ambroise-Tho mas P.Vaccination against malaria. D isappointments and hopes. Bull Acad N atl Med 1997;181(8):1637-1650.

51.Taylor-Robinson A.Vaccination against malaria:Targets, strategies and potentiation of immunity to blood stage parasites. Front Biosci 2000;5:16-29.
52. Scherf A, Behr C, Sarthou JL, Pla M, Rogier C, Trape JF et al. Immune response in mouse and malaria-exposed humans to peptides derived from Pfl1-1, a highly repetitive megadalton protein of Plasmodium falciparum. Eur J Immunol 1993;23:1574-1581.

53. Baker DA,Thompson J, Daramola 0, C arlton JM, Targett G. Sexual stage specific RN A expresssion of a new Plasmodium falciparum gene detected by in situ hybridization. Mol Biochem Parasitol 1995;72:193-201. 54. Premawansa S, Gamage-Mendis A, Perera L, Begarnie S, Mendis K, Carter R. Plasmodium falciparum malaria transmission-blocking immunity under conditions of low endemicity as in Sri Lanka. Parasite Immunol 1994;16:35-42.

55. D uffy PE, Kaslow DC . A novel malaria protein, Pfs 28 and Pfs 25 are genetically linked and synergistic as falciparum malaria transmissionblocking vaccines. Infect Immun 1997;65:1109-1113.

56. Hisaeda H, Stowers A, T suboi T, Collins W, Sattabongkot J, Suwanabun $N$ et al. Antibodies to malaria vaccine candidates Pvs 25 and Pvs28 completely block the ability of Plasmodium vivax to infect mosquitoes. Infect Immun 2000;68:6618-6623.

57. Silvestrini F,Alano P,W illiams J. Commitment to the production of male and female gametocytes in the human malaria parasite Plasmodium falciparum. Parasitology 2000;121(Pt 5):465-471.

58. Eksi S,W illiamson KC. Male-specific expression of the paralog of malaria transmissionblocking target antigen Pfs230, PfB0400w. Mol Biochem Parasitol 2002;122(2):127-30.

59. Piper K, Hayward R, Cox M, D ay K. Malaria transmission and naturally acquired immunity to PfEMP-1. Infect Immun 1999;67:6369-6374. 60.Abdel-W ahab A,A bdel-Muhsin AM, Ali E, Suleiman S,A hmed S, $W$ alliker $D$ et al. Dynamics of gametocytes among Plasmodium falciparum clones in natural infections in an area of highly seasonal transmission.J Infect D is 2002;185(12):1838-1842.

61. D elrieu I,W aller CC, Mota MM, G rainger M, Langhorne J, Holder AA. PSLAP, a protein with multiple adhesive motifs, is expressed in Plasmodium falciparum gametocytes. Mol Biochem Parasito 1 2002;121(1):11-20.

62. Healer J, Graszynski A, Riley EM. Phagocytosis does not play a mayor role naturally acquired transmission-blocking immunity to Plasmodium falciparum malaria. Infect Immun 1999;67(5):2334-2339. 